Article

\title{
Some Cosmological Solutions of a New Nonlocal Gravity Model
}

\author{
Ivan Dimitrijevic ${ }^{1, *}$, Branko Dragovich ${ }^{2,3}$, Alexey S. Koshelev ${ }^{4}$, Zoran Rakic ${ }^{1}$ \\ and Jelena Stankovic 5 \\ 1 Faculty of Mathematics, University of Belgrade, Studentski trg 16, 11158 Belgrade, Serbia; \\ zrakic@matf.bg.ac.rs \\ 2 Institute of Physics, University of Belgrade, 11080 Belgrade, Serbia; dragovich@ipb.ac.rs \\ 3 Mathematical Institute, Serbian Academy of Sciences and Arts, 11000 Belgrade, Serbia \\ 4 Departamento de Física and Centro de Matemática e Aplicações, Universidade da Beira Interior, \\ 6200 Covilhã, Portugal; alexey@ubi.pt \\ 5 Teacher Education Faculty, University of Belgrade, Kraljice Natalije 43, 11000 Belgrade, Serbia; \\ jelena.grujic@uf.bg.ac.rs \\ * Correspondence: ivand@matf.bg.ac.rs
}

Received: 28 April 2020; Accepted: 20 May 2020; Published: 2 June 2020

check for updates

\begin{abstract}
In this paper, we investigate a nonlocal modification of general relativity (GR) with action $S=\frac{1}{16 \pi G} \int[R-2 \Lambda+(R-4 \Lambda) \mathcal{F}(\square)(R-4 \Lambda)] \sqrt{-g} d^{4} x$, where $\mathcal{F}(\square)=\sum_{n=1}^{+\infty} f_{n} \square^{n}$ is an analytic function of the d'Alembertian $\square$. We found a few exact cosmological solutions of the corresponding equations of motion. There are two solutions which are valid only if $\Lambda \neq 0, k=0$, and they have no analogs in Einstein's gravity with cosmological constant $\Lambda$. One of these two solutions is $a(t)=A \sqrt{t} e^{\frac{\Lambda}{4}} t^{2}$, that mimics properties similar to an interference between the radiation and the dark energy. Another solution is a nonsingular bounce one $a(t)=A e^{\Lambda t^{2}}$. For these two solutions, some cosmological aspects are discussed. We also found explicit form of the nonlocal operator $\mathcal{F}(\square)$, which satisfies obtained necessary conditions.
\end{abstract}

Keywords: nonlocal gravity; cosmological solutions; radiation; dark energy

\section{Introduction}

General relativity [1], or in other words Einstein theory of gravity, is recognized as one of the best physical theories - with beautiful theoretical properties and significant phenomenological achievements. General relativity (GR) very well describes dynamics of the Solar System. It predicted several important phenomena that were confirmed: deflection of light by the Sun, gravitational light redshift, gravitational waves, gravitational lensing, and black holes.

Despite its extraordinary success, GR should not be viewed as a final theory of gravity. For example, from the standard cosmological model, which assumes applicability of GR to the universe as a whole, follows that the universe is approximately made of $68 \%$ of dark energy (DE), $27 \%$ of dark matter (DM), and only 5\% of visible (standard) matter. However, DE and DM are not yet experimentally detected, and validity of GR at very large cosmic scales is not confirmed. Even in the case of discovering DM and DE, there is still a sense to look for modifications of GR that may mimic the same or similar effects as those of DE and DM. In addition, cosmological solutions of GR, under rather general properties of the matter, contain singularity at the cosmic time $t=0$. In addition to these mentioned astrophysical and cosmological problems, there are also some problems that are pure theoretical and come from quantum gravity and string theory. Note also that there is no known reliable theoretical principle that might show the right direction for valuable extension of GR. As a result of all these 
shortages, there are many approaches towards possible generalization of Einstein's theory of gravity (for a review, see [2-6]).

One of the current ways towards modification of GR is nonlocal modified gravity, see e.g., [7-24]. All nonlocal gravity models contain the d'Alembert-Beltrami operator $\square$, that is involved mainly in two ways: (1) in the form $\square^{-n}$ and (2) as an analytic function $F(\square)=\sum_{n=0}^{+\infty} f_{n} \square^{n}$. Models with $\square^{-n}$ operator are introduced to investigate the late cosmic time acceleration without its matter origin. Some of such models are given by the action

$$
S=\frac{1}{16 \pi G} \int \sqrt{-g}(R+\mathcal{L}) d^{4} x,
$$

where $\mathcal{L}=R f\left(\square^{-1} R\right)$ (see, e.g., [4,8,24]), and $\mathcal{L}=-\frac{1}{6} m^{2} R \square^{-2} R$ (see [9] and references therein).

An interesting and promising class of nonlocal gravity models, that have been recently considered, is given by

$$
S=\frac{1}{16 \pi G} \int_{\mathcal{M}} \sqrt{-g}[R-2 \Lambda+P(R) \mathcal{F}(\square) Q(R)] d^{4} x,
$$

where $\mathcal{M}$ is a pseudo-Riemannian manifold of signature $(1,3)$ with metric $\left(g_{\mu v}\right), \Lambda$ is the cosmological constant, $P(R)$ and $Q(R)$ are some differentiable functions of the Ricci scalar $R$, and $\mathcal{F}(\square)=\sum_{n=1}^{+\infty} f_{n} \square^{n}$. Motivation to use this analytic nonlocal operator comes from ordinary and $p$-adic string theory (see [25] and references therein) and observation that some analytic nonlocal operators may improve renormalizability in some quantum gravity models, see [26-28]. To have better insight into effects, preliminary investigation of these models is usually without matter.

Note again that Equation (2) contains a class of simple nonlocal extensions of GR, but still in rather general form. Usually researchers start by a particular expression for $P(R)$ and $Q(R)$ as differentiable functions of $R$, while $\mathcal{F}(\square)$ is treated as an analytic function of operator $\square$, whose concrete form is not given at the beginning. For given $P(R)$ and $Q(R)$, the next step is derivation of equations of motion for metric tensor $g_{\mu v}$. To consider (2) as nonlocal gravity model of interest for cosmology, equations of motion should have some useful cosmological solutions. Existence of such (usually exact) cosmological solutions requires some restrictions on the function $\mathcal{F}(\square)=\sum_{n=1}^{+\infty} f_{n} \square^{n}$, i.e., on its coefficients $f_{n}$, e.g., see [10-14]. Then with these, and perhaps some additional, restrictions there is a possibility to construct the corresponding concrete function $\mathcal{F}(\square)$. Since we do not know a priori function $\mathcal{F}(\square)$, this approach is a reasonable way to get it.

Concerning Equation (2), the most attention has been paid to the simple case when $P(R)=$ $Q(R)=R$, e.g., see $[10,11,17,18,20,29-36]$. This investigation started in $[10,11]$ by successful attempt to find nonsingular bouncing solution of the Big Bang singularity problem in standard cosmology. To find appropriate solution of equations of motion, the ansatz $\square R=r R+s$ was used, where $r$ and $s$ are parameters that connect the solution and function $\mathcal{F}(\square)$. If also in this case cosmological constant $\Lambda=0$ then it is some kind of nonlocal generalization of the Starobinsky $R^{2}$ inflation model, whose various properties are studied in $[19,20]$.

Another very intriguing example of the nonlocal gravity in Equation (2) has $P(R)=Q(R)=$ $\sqrt{R-2 \Lambda}$ [37]. One of its exact cosmological solutions is $a(t)=A t^{\frac{2}{3}} e^{\frac{\Lambda}{14}} t^{2}, \Lambda \neq 0, k=0$. This solution mimics properties similar to an interplay of the dark matter and the dark energy. Moreover, computed cosmological parameters are in a good agreement with astronomical observations. It is worth noting that at the first glance appearance of $\sqrt{R-2 \Lambda}$ in this model may look strange. However, it can be regarded as a natural nonlocal generalization of the standard local Lagrangian $R-2 \Lambda$. Namely, one can introduce nonlocality as follows: $R-2 \Lambda=\sqrt{R-2 \Lambda} \sqrt{R-2 \Lambda} \rightarrow \sqrt{R-2 \Lambda}[1+\mathcal{F}(\square)] \sqrt{R-2 \Lambda}$.

Nonlocal gravity model which we investigate in this paper has $P(R)=Q(R)=R-4 \Lambda$, and the action is given explicitly below in Equation (3). As we will see, one of the exact cosmological solutions is $a(t)=A \sqrt{t} e^{\frac{\Lambda}{4}} t^{2}, \Lambda \neq 0, k=0$, which mimics an interplay between radiation and the dark energy. Nonlocal term $(R-4 \Lambda) \mathcal{F}(\square)(R-4 \Lambda)$ in this model arose in the process of generalization of the above 
mentioned model with nonlocality $R \mathcal{F}(\square) R$. The starting expression was $\left(R-R_{0}\right) \mathcal{F}(\square)\left(R-R_{0}\right)$, where $R_{0}$ is a constant that may lead to some interesting background solutions.

Section 2 contains derivation of the equations of motion. Section 3 is devoted to the exact cosmological solutions. Some concluding remarks are in Section 4.

\section{New Nonlocal Gravity Model}

The action of our nonlocal gravity model is

$$
S=\frac{1}{16 \pi G} \int[R-2 \Lambda+(R-4 \Lambda) \mathcal{F}(\square)(R-4 \Lambda)] \sqrt{-g} d^{4} x
$$

where $\mathcal{F}(\square)=\sum_{n=1}^{\infty} f_{n} \square^{n}$ and $\square=\nabla_{\mu} \nabla^{\mu}=\frac{1}{\sqrt{-g}} \partial_{\mu}\left(\sqrt{-g} g^{\mu v} \partial_{\nu}\right)$ is the corresponding d'Alembert-Beltrami operator. In construction of Equation (3) we started from action

$$
S=\frac{1}{16 \pi G} \int\left[R-2 \Lambda+\left(R-R_{0}\right) \mathcal{F}(\square)\left(R-R_{0}\right)\right] \sqrt{-g} d^{4} x
$$

and found that for $R_{0}=4 \Lambda$ the corresponding equations of motion, Equations (13) and (14), give two interesting background solutions presented in Sections 3.1 and 3.2.

\subsection{Equations of Motion}

The equations of motion for nonlocal gravity action in Equation (2) are derived in [38] and have the following form:

$$
\hat{G}_{\mu v}=G_{\mu v}+\Lambda g_{\mu \nu}-\frac{1}{2} g_{\mu \nu} P(R) \mathcal{F}(\square) Q(R)+\left(R_{\mu v}-K_{\mu \nu}\right) W+\frac{1}{2} \Omega_{\mu v}=0,
$$

where $\hat{G}_{\mu \nu}$ is nonlocal version of Einstein's tensor, and

$$
\begin{aligned}
K_{\mu v} & =\nabla_{\mu} \nabla_{v}-g_{\mu v} \square, \\
W & =P^{\prime}(R) \mathcal{F}(\square) Q(R)+Q^{\prime}(R) \mathcal{F}(\square) P(R), \\
\Omega_{\mu v} & =\sum_{n=1}^{\infty} f_{n} \sum_{\ell=0}^{n-1} S_{\mu v}\left(\square^{\ell} P(R), \square^{n-1-\ell} Q(R)\right), \\
S_{\mu v}(A, B) & =g_{\mu \nu} \nabla^{\alpha} A \nabla_{\alpha} B+g_{\mu v} A \square B-2 \nabla_{\mu} A \nabla_{\nu} B,
\end{aligned}
$$

where $P^{\prime}(R)$ and $Q^{\prime}(R)$ are derivatives of $P(R)$ and $Q(R)$ with respect to R, respectively.

From computation in detail, it follows

$$
\nabla^{\mu} \hat{G}_{\mu v}=0 .
$$

Equation (3) is a particular case of Equation (2) and the corresponding equations of motion for model in Equation (3) easily follow from Equation (5), i.e., equations of motion are

$$
\begin{aligned}
& \hat{G}_{\mu v}=G_{\mu v}+\Lambda g_{\mu \nu}-\frac{1}{2} g_{\mu \nu} U \mathcal{F}(\square) U+2\left(R_{\mu v}-\nabla_{\mu} \nabla_{v}+g_{\mu \nu} \square\right) \mathcal{F}(\square) U \\
& +\frac{1}{2} \sum_{n=1}^{+\infty} f_{n} \sum_{\ell=0}^{n-1}\left(g_{\mu v}\left(g^{\alpha \beta} \partial_{\alpha} \square^{\ell} U \partial_{\beta} \square^{n-1-\ell} U+\square^{\ell} U \square^{n-\ell} U\right)\right. \\
& \left.-2 \partial_{\mu} \square^{\ell} U \partial_{\nu} \square^{n-1-\ell} U\right)=0,
\end{aligned}
$$

where $G_{\mu v}=R_{\mu \nu}-\frac{1}{2} R g_{\mu \nu}$ is the Einstein tensor, and $U=R-4 \Lambda$. 
In the sequel of this paper, we are mainly interested in finding and investigating some exact cosmological solutions of Equation (11). Since the universe is homogeneous and isotropic at large scales, it has the Friedmann-Lemaître-Robertson-Walker (FLRW) metric

$$
d s^{2}=-d t^{2}+a^{2}(t)\left(\frac{d r^{2}}{1-k r^{2}}+r^{2} d \theta^{2}+r^{2} \sin ^{2} \theta d \phi^{2}\right), \quad(c=1), k=0, \pm 1,
$$

where $a(t)$ is the cosmic scale factor. As a consequence of symmetries of the FLRW metric, Equation (11) can be reduced to two independent differential equations and we take trace and 00-component, respectively:

$$
\begin{aligned}
& 4 \Lambda-R-2 U \mathcal{F}(\square) U+2(R+3 \square) \mathcal{F}(\square) U \\
& +\sum_{n=1}^{+\infty} f_{n} \sum_{\ell=0}^{n-1}\left(\partial_{\alpha} \square^{\ell} U \partial^{\alpha} \square^{n-1-\ell} U+2 \square^{\ell} U \square^{n-\ell} U\right)=0, \\
& G_{00}-\Lambda+\frac{1}{2} U \mathcal{F}(\square) U+2\left(R_{00}-\partial_{0} \partial_{0}-\square\right) \mathcal{F}(\square) U \\
& -\frac{1}{2} \sum_{n=1}^{+\infty} f_{n} \sum_{\ell=0}^{n-1}\left(\partial_{\alpha} \square^{\ell} U \partial^{\alpha} \square^{n-1-\ell} U+\square^{\ell} U \square^{n-\ell} U\right. \\
& \left.+2 \partial_{0} \square^{\ell} U \partial_{0} \square^{n-1-\ell} U\right)=0,
\end{aligned}
$$

where

$$
R_{00}=-3 \frac{\ddot{a}}{a}, \quad G_{00}=3 \frac{\dot{a}^{2}+k}{a^{2}} .
$$

Equation (11) can be rewritten in the form

$$
\hat{G}_{\mu v}=G_{\mu v}+\Lambda g_{\mu \nu}-8 \pi G \hat{T}_{\mu \nu}=0,
$$

where $\hat{T}_{\mu \nu}$ can be regarded as a nonlocal gravity analog of the energy-momentum tensor in Einstein's gravity. The corresponding Friedmann equations to Equation (16) are

$$
\frac{\ddot{a}}{a}=-\frac{4 \pi G}{3}(\bar{\rho}+3 \bar{p})+\frac{\Lambda}{3}, \quad \frac{\dot{a}^{2}+k}{a^{2}}=\frac{8 \pi G}{3} \bar{\rho}+\frac{\Lambda}{3},
$$

where $\bar{\rho}$ and $\bar{p}$ play a role of the energy density and pressure of the dark side of the universe, respectively. The related equation of state is

$$
\bar{p}(t)=\bar{w}(t) \bar{\rho}(t) .
$$

\subsection{Ghost-Free Condition}

The spectrum can be found and a possibility to avoid ghost degrees of freedom can be studied by considering the second variation of the action. This task was accomplished in different settings. In paper [15], it was done for an action which contains our action in Equation (3) as one of the terms. In paper [32] analogous analysis was performed for generic functions $P(R)$ and $Q(R)$. The generic idea is that certain combinations containing the operator function $\mathcal{F}(\square)$ form kinetic operators for scalar and tensor propagating degrees of freedom. Consequently such combinations must be equal to an exponent of an entire function. The latter has no zeros on the whole complex plane and as such does not result in poles in propagators yielding no new degrees of freedom. Detailed expressions and all the restrictions can be found in the above mentioned references. 


\section{Cosmological Solutions}

Our intention is to obtain some exact cosmological solutions of the equations of motion in Equations (13) and (14) in the form $a(t)=A t^{m} e^{\gamma t^{2}}$, where $m$ and $\gamma$ are some constants. At the beginning we take $U=R-R_{0}$ and $k=0$ in the equations of motion. Thus we have three parameters $m, \gamma, R_{0}$ that have to be determined. We found that for $R_{0}=4 \Lambda$ there are two pairs of solutions for $m$ and $\gamma$ : (1) $m=\frac{1}{2}, \gamma=\frac{\Lambda}{4}$ and (2) $m=0, \gamma=\Lambda$. These background solutions are presented below.

Recall that scalar curvature for the FLRW metric in Equation (12) is

$$
R(t)=6\left(\frac{\ddot{a}}{a}+\left(\frac{\dot{a}}{a}\right)^{2}+\frac{k}{a^{2}}\right) .
$$

The d'Alembert-Beltrami operator $\square$ acts as $\square R=-\frac{\partial^{2}}{\partial t^{2}} R-3 H \frac{\partial}{\partial t} R$, where $H=\frac{\dot{a}}{a}$ is the Hubble parameter.

Note that the Minkowski space $(\mathrm{a}(\mathrm{t})=$ const., $R=\Lambda=k=0)$ is a solution of equations of motion (13) and (14).

In what follows, we will present and briefly discuss some exact cosmological solutions mainly with $\Lambda \neq 0$.

3.1. Cosmological Solution $a(t)=A \sqrt{t} e^{\frac{\Lambda}{4} t^{2}}, k=0$

For this solution we have

$$
\dot{a}(t)=a(t) \frac{1}{2}\left(t^{-1}+\Lambda t\right), \quad \ddot{a}(t)=a(t) \frac{1}{4}\left(\Lambda^{2} t^{2}+4 \Lambda-t^{-2}\right),
$$

and scalar curvature in Equation (19) becomes

$$
R(t)=3 \Lambda\left(\Lambda t^{2}+3\right) .
$$

The Hubble parameter is

$$
H(t)=\frac{1}{2}\left(t^{-1}+\Lambda t\right)
$$

and its first part $\left(\frac{1}{2 t}\right)$ is the same as for the radiation dominance in Einstein's gravity, while the second term $\left(\frac{\Lambda t}{2}\right)$ can be related to the dark energy generated by cosmological constant $\Lambda$. It is evident that this dark radiation is dominated at the small cosmic times and can be ignored compared to $\Lambda$ term at large times. At the present cosmic time $t_{0}=13.801 \times 10^{9}$ year and $\Lambda=0.98 \times 10^{-35} \mathrm{~s}^{-2}$, both terms in Equation (22) are of the same order of magnitude and $H\left(t_{0}\right)=100.2 \mathrm{~km} / \mathrm{s} / \mathrm{Mpc}$. This value for the Hubble parameter is larger than current Planck mission result $H_{0}=(67.40 \pm 0.50) \mathrm{km} / \mathrm{s} / \mathrm{Mpc}[39]$. Hence this cosmological solution may be of interest for the early universe with radiation dominance and for far-future accelerated expansion.

There is useful equality

$$
\square(R-4 \Lambda)=-3 \Lambda(R-4 \Lambda)
$$

which leads to

$$
\mathcal{F}(\square)(R-4 \Lambda)=\mathcal{F}(-3 \Lambda)(R-4 \Lambda) .
$$

$R_{00}$ and $G_{00}$ are:

$$
R_{00}=\frac{3}{4}\left(t^{-2}-4 \Lambda-\Lambda^{2} t^{2}\right), \quad G_{00}=\frac{3}{4}\left(t^{-1}+\Lambda t\right)^{2} .
$$


Using equality $\square(R-4 \Lambda)=-3 \Lambda(R-4 \Lambda)$, the trace Equation (13) becomes

$$
4 \Lambda-R-10 \Lambda(R-4 \Lambda) \mathcal{F}(-3 \Lambda)+\left(-6 \Lambda(R-4 \Lambda)^{2}-\dot{R}^{2}\right) \mathcal{F}^{\prime}(-3 \Lambda)=0 .
$$

The 00 component of EOM in Equation (14) becomes

$$
\begin{aligned}
& G_{00}-\Lambda+(R-4 \Lambda) \mathcal{F}(-3 \Lambda)\left(2 R_{00}+\frac{1}{2} R+4 \Lambda\right)-2 \mathcal{F}(-3 \Lambda) \ddot{R} \\
& +\frac{1}{2}\left(3 \Lambda(R-4 \Lambda)^{2}-\dot{R}^{2}\right) \mathcal{F}^{\prime}(-3 \Lambda)=0 .
\end{aligned}
$$

Substituting scalar curvature $R$ into the trace Equation (26) we obtain

$$
\begin{aligned}
& 4 \Lambda-3 \Lambda\left(3+\Lambda t^{2}\right)-10 \Lambda^{2}\left(5+3 \Lambda t^{2}\right) \mathcal{F}(-3 \Lambda) \\
& +\left(-6 \Lambda^{3}\left(9 \Lambda^{2} t^{4}+30 \Lambda t^{2}+25\right)-36 \Lambda^{4} t^{2}\right) \mathcal{F}^{\prime}(-3 \Lambda)=0 .
\end{aligned}
$$

Similarly, the 00 component of EOM in Equation (27) becomes

$$
\begin{aligned}
& \frac{3\left(1+\Lambda t^{2}\right)^{2}}{4 t^{2}}-\Lambda+\Lambda\left(5+3 \Lambda t^{2}\right)\left(\frac{3}{2 t^{2}}+\frac{5}{2} \Lambda\right) \mathcal{F}(-3 \Lambda) \\
& -12 \Lambda^{2} \mathcal{F}(-3 \Lambda)+\frac{1}{2}\left(3 \Lambda^{3}\left(9 \Lambda^{2} t^{4}+30 \Lambda t^{2}+25\right)-36 \Lambda^{4} t^{2}\right) \mathcal{F}^{\prime}(-3 \Lambda)=0 .
\end{aligned}
$$

Finally, the solution of equations of motion in Equations (13) and (14) requires constraints

$$
\mathcal{F}(-3 \Lambda)=-\frac{1}{10 \Lambda}, \quad \mathcal{F}^{\prime}(-3 \Lambda)=0, \quad \Lambda \neq 0,
$$

which are satisfied by nonlocal operator

$$
\mathcal{F}(\square)=\frac{\square}{30 \Lambda^{2}} \exp \left(\frac{\square}{3 \Lambda}+1\right)
$$

From Equation (17) follows

$$
\bar{\rho}(t)=\frac{3 t^{-2}+3 \Lambda^{2} t^{2}+2 \Lambda}{32 \pi G}, \quad \bar{p}(t)=\frac{t^{-2}-3 \Lambda^{2} t^{2}-6 \Lambda}{32 \pi G} .
$$

One can easily conclude that

$$
\bar{w}=\frac{t^{-2}-3 \Lambda^{2} t^{2}-6 \Lambda}{3 t^{-2}+3 \Lambda^{2} t^{2}+2 \Lambda} \rightarrow \begin{cases}-1, & t \rightarrow \infty \\ \frac{1}{3}, & t \rightarrow 0 .\end{cases}
$$

From Equation (33), we see that parameter $\bar{w}$ behaves: (i) like $1 / 3$ at early times as for the case of radiation and (ii) like -1 as in the usual prediction for the late times acceleration with cosmological constant $\Lambda$.

3.2. Cosmological Solution $a(t)=A e^{\Lambda t^{2}}, k=0$

For this solution we have

$$
\begin{aligned}
& \dot{a}(t)=a(t) 2 \Lambda t, \quad \ddot{a}(t)=a(t) 2 \Lambda\left(2 \Lambda t^{2}+1\right), \\
& R(t)=12 \Lambda\left(4 \Lambda t^{2}+1\right), \quad H(t)=2 \Lambda t, \\
& R_{00}=-6 \Lambda\left(1+2 \Lambda t^{2}\right), \quad G_{00}=12 \Lambda^{2} t^{2} .
\end{aligned}
$$

There are useful equalities:

$$
\square(R-4 \Lambda)=-12 \Lambda(R-4 \Lambda), \quad \mathcal{F}(\square)(R-4 \Lambda)=\mathcal{F}(-12 \Lambda)(R-4 \Lambda) .
$$


Using the equalities in Equation (37), the trace Equation (13) becomes

$$
4 \Lambda-R-64 \Lambda(R-4 \Lambda) \mathcal{F}(-12 \Lambda)+\left(-24 \Lambda(R-4 \Lambda)^{2}-\dot{R}^{2}\right) \mathcal{F}^{\prime}(-12 \Lambda)=0 .
$$

The 00 component of EOM in Equation (14) is as follows:

$$
\begin{aligned}
& G_{00}-\Lambda+(R-4 \Lambda) \mathcal{F}(-12 \Lambda)\left(2 R_{00}+\frac{1}{2} R+22 \Lambda\right)-2 \mathcal{F}(-12 \Lambda) \ddot{R} \\
& +\frac{1}{2}\left(12 \Lambda(R-4 \Lambda)^{2}-\dot{R}^{2}\right) \mathcal{F}^{\prime}(-12 \Lambda)=0 .
\end{aligned}
$$

Substituting scalar curvature $R$ in Equation (35) into the trace Equation (38) we obtain

$$
\begin{aligned}
& 4 \Lambda-12 \Lambda\left(1+4 \Lambda t^{2}\right)-512 \Lambda^{2}\left(1+6 \Lambda t^{2}\right) \mathcal{F}(-12 \Lambda) \\
& +\left(-1536 \Lambda^{3}\left(36 \Lambda^{2} t^{4}+12 \Lambda t^{2}+1\right)-9216 \Lambda^{4} t^{2}\right) \mathcal{F}^{\prime}(-12 \Lambda)=0 .
\end{aligned}
$$

Similarly, the 00 component of EOM in Equation (39) becomes

$$
\begin{aligned}
& 12 \Lambda^{2} t^{2}-\Lambda+8 \Lambda\left(1+6 \Lambda t^{2}\right)\left(2\left(-12 \Lambda^{2} t^{2}-6 \Lambda\right)+6 \Lambda\left(1+4 \Lambda t^{2}\right)+22 \Lambda\right) \mathcal{F}(-12 \Lambda) \\
& -2 \mathcal{F}(-12 \Lambda) 96 \Lambda^{2}+\frac{1}{2}\left(768 \Lambda^{3}\left(36 \Lambda^{2} t^{4}+12 \Lambda t^{2}+1\right)-9216 \Lambda^{4} t^{2}\right) \mathcal{F}^{\prime}(-12 \Lambda)=0 .
\end{aligned}
$$

To be satisfied, equations of motion in Equations (13) and (14) imply conditions

$$
\mathcal{F}(-12 \Lambda)=-\frac{1}{64 \Lambda}, \quad \mathcal{F}^{\prime}(-12 \Lambda)=0, \quad \Lambda \neq 0,
$$

that can be realized by

$$
\mathcal{F}(\square)=\frac{\square}{768 \Lambda^{2}} \exp \left(\frac{\square}{12 \Lambda}+1\right) .
$$

According to Equation (17) follows

$$
\bar{\rho}(t)=\frac{\Lambda\left(12 \Lambda t^{2}-1\right)}{8 \pi G}, \quad \bar{p}(t)=-\frac{3 \Lambda\left(4 \Lambda t^{2}+1\right)}{8 \pi G} .
$$

The corresponding $\bar{w}$ parameter is

$$
\bar{w}=\frac{-12 \Lambda t^{2}-3}{12 \Lambda t^{2}-1} \rightarrow \begin{cases}-1, & t \rightarrow \infty \\ 3, & t \rightarrow 0\end{cases}
$$

\subsection{Other Vacuum Solutions: $R(t)=$ const}

The above two cosmological solutions have scalar curvature $R(t)$ dependent on time $t$. There are also vacuum solutions with $R=4 \Lambda$ that are the same as for Einstein's equations of motion. Since $\square(R-$ $4 \Lambda)=0$, it is evident that such solutions satisfy equations of motion in Equations (13) and (14) without conditions on function $\mathcal{F}(\square)$.

In addition to the already mentioned Minkowski space, there is another solution with $R(t)=0$ :

Milne solution: $a(t)=t, k=-1, \Lambda=0,(c=1)$.

From our analysis follows that there are no other exact power law solutions of the form $a(t)=A t^{\alpha}$ except this Milne one. 


\section{Concluding Remarks}

In this article, we have presented some exact cosmological solutions of nonlocal gravity model without matter given by Equation (3). Two of these solutions are valid only if $\Lambda \neq 0$. The solutions $a(t)=A \sqrt{t} e^{\frac{\Lambda}{4}} t^{2}$ and $a(t)=A e^{\Lambda t^{2}}$ are not contained in Einstein's gravity with cosmological constant $\Lambda$. The solution $a(t)=A \sqrt{t} e^{\frac{\Lambda}{4} t^{2}}$ mimics interference between expansion with radiation $a(t)=A \sqrt{t}$ and a dark energy $a(t)=A e^{\frac{\Lambda}{4} t^{2}}$.

The solution $a(t)=A e^{\Lambda t^{2}}$ is a nonsingular bounce one and an even function of cosmic time. An exact cosmological solution of the type $a(t)=A e^{\alpha \Lambda t^{2}}$, where $\alpha$ is a number, appears also at least in the following two models of Equation (2): (1) $P(R)=Q(R)=R$ [17] (see also [18]), and (2) $P(R)=Q(R)=\sqrt{R-2 \Lambda}$ [37]. It would be interesting to investigate other possible models with this kind of solution.

With respect to the cosmological solutions $a(t)=A \sqrt{t} e^{\frac{\Lambda}{4} t^{2}}$ and $a(t)=A e^{\Lambda t^{2}}$, the nonlocal analytic operator $\mathcal{F}(\square)$ is presented by Equations (31) and (43), respectively. Operator $\mathcal{F}(\square)$ that takes into account both solutions should have the form $\mathcal{F}(\square)=a \frac{u}{\Lambda} \exp \left(b u^{3}+c u^{2}+d u\right)$, where $a, b, c, d$, are some definite constants and $u=\square / \Lambda$ is dimensionless operator. We do not introduce an additional parameter like mass $M$.

According to our solutions $a(t)=A \sqrt{t} e^{\frac{\Lambda}{4}} t^{2}$ and $a(t)=A t^{\frac{2}{3}} e^{\frac{\Lambda}{14} t^{2}}$ [37], it follows that effects of the dark radiation $(\sqrt{t})$, the dark matter $\left(t^{\frac{2}{3}}\right)$ and the dark energy $\left(e^{\alpha \Lambda t^{2}}\right)$ at the cosmic scale can be generated by suitable nonlocal gravity models. These findings should play useful role in further research concerning the universe evolution.

Author Contributions: Conceptualization, I.D., B.D., A.S.K., Z.R., and J.S.; methodology, I.D., B.D., A.S.K., Z.R., and J.S.; investigation, I.D., B.D., A.S.K., Z.R., and J.S.; writing-original draft preparation, B.D.; writing-review and editing, I.D., B.D., A.S.K., Z.R., and J.S. All authors have read and agreed to the published version of the manuscript.

Funding: This research is partially supported by the Ministry of Education, Science and Technological Development of Republic of Serbia, grant No. 174012.

Acknowledgments: The authors would like to thank the reviewers for their comments and suggestions that resulted in improvement of paper presentation.

Conflicts of Interest: The authors declare no conflict of interest.

\section{References}

1. Wald, R.M. General Relativity; University of Chicago Press: Chicago, IL, USA, 1984.

2. Sotiriou, T.P.; Faraoni, V. $f(R)$ theories of gravity. Rev. Mod. Phys. 2010, 82, 451-497. [CrossRef]

3. Clifton, T.; Ferreira, P.G.; Padilla, A.; Skordis, C. Modified gravity and cosmology. Phys. Rep. 2012, 513, 1-189. [CrossRef]

4. Nojiri, S.; Odintsov, S.D. Unified cosmic history in modified gravity: From $F(R)$ theory to Lorentz non-invariant models. Phys. Rep. 2011, 505, 59-144. [CrossRef]

5. Nojiri, S.; Odintsov, S.D.; Oikonomou, V.K. Modified gravity theories on a nutshell: Inflation, bounce and late-time evolution. Phys. Rep. 2017, 692, 1-104. [CrossRef]

6. Novello, M.; Bergliaffa, S.E.P. Bouncing cosmologies. Phys. Rep. 2008, 463, 127-213. [CrossRef]

7. Deser, S.; Woodard, R. Nonlocal cosmology. Phys. Rev. Lett. 2007, 99, 111-301. [CrossRef]

8. Woodard, R.P. Nonlocal models of cosmic acceleration. Found. Phys. 2014, 44, 213-233. [CrossRef]

9. Belgacem, E.; Dirian, Y.; Foffa, S.; Maggiore, M. Nonlocal gravity. Conceptual aspects and cosmological predictions. J. Cosmol. Astropart. Phys. 2018, 2018, 2. [CrossRef]

10. Biswas, T.; Mazumdar, A.; Siegel, W. Bouncing universes in string-inspired gravity. J. Cosmol. Astropart. Phys. 2006, 603, 9. [CrossRef]

11. Biswas, T.; Koivisto, T.; Mazumdar, A. Towards a resolution of the cosmological singularity in non-local higher derivative theories of gravity. J. Cosmol. Astropart. Phys. 2010, 1011, 8. [CrossRef]

12. Biswas, T.; Gerwick, E.; Koivisto, T.; Mazumdar, A. Towards singularity and ghost free theories of gravity. Phys. Rev. Lett. 2012, 108, 31-101. [CrossRef] [PubMed] 
13. Biswas, T.; Koshelev, A.S.; Mazumdar, A.; Vernov, S. Yu. Stable bounce and inflation in non-local higher derivative cosmology. J. Cosmol. Astropart. Phys. 2012, 8, 24. [CrossRef]

14. Biswas, T.; Conroy, A.; Koshelev, A.S.; Mazumdar, A. Generalized gost-free quadratic curvature gravity. Class. Quantum Grav. 2014, 31, 159501. [CrossRef]

15. Biswas, T.; Koshelev, A.S.; Mazumdar, A. Consistent higher derivative gravitational theories with stable de Sitter and Anti-de Sitter backgrounds. Phys. Rev. 2017, 95, 043533. [CrossRef]

16. Dragovich, B. On Nonlocal modified gravity and cosmology. Springer Proc. Math. Stat. 2014, 111, $251-262$.

17. Koshelev, A.S.; Vernov, S.Y. On bouncing solutions in non-local gravity. Phys. Part. Nuclei 2012, 43, 666-668. [CrossRef]

18. Koshelev, A.S. Stable analytic bounce in non-local Einstein-Gauss-Bonnet cosmology. Class. Quantum Grav. 2013, 30, 155001. [CrossRef]

19. Koshelev, A.S.; Kumar, K.S.; Starobinsky, A.A. $R^{2}$ inflation to probe non-perturbative quantum gravity. JHEP 2018, 1803, 71. [CrossRef]

20. Koshelev, A.S.; Modesto, L.; Rachwal, L.; Starobinsky, A.A. Occurrence of exact $R^{2}$ inflation in non-local UV-complete gravity. JHEP 2016, 11, 1-41.

21. Buoninfante, L.; Koshelev, A. S.; Lambiase, G.; Mazumdar, A. Classical properties of non-local, ghost- and singularity-free gravity. JCAP 2018, 2018, 34. [CrossRef]

22. Koshelev, A.S.; Marto, J.; Mazumdar, A. Towards conformally flat, non-Kasner vacuum solution in infinite derivative gravity. JCAP 2019, 2019, 20. [CrossRef]

23. Elizalde, E.; Pozdeeva, E.O.; Vernov, S.Y. Stability of de Sitter solutions in non-local cosmological models. arXiv 2013, arXiv:1202.0178.

24. Conroy, A.; Koivisto, T.; Mazumdar, A.; Teimouri, A. Generalised quadratic curvature, non-local infrared modifications of gravity and Newtonian potentials. Clas. Quantum Grav. 2015, 32, 015024. [CrossRef]

25. Dragovich, B.; Khrennikov, A.Y.; Kozyrev, S.V.; Volovich, I.V.; Zelenov, E.I. p-Adic mathematical physics: The first 30 years. p-Adic Numbers Ultrametric Anal. Appl. 2017, 9, 87-121. [CrossRef]

26. Modesto, L. Super-renormalizable quantum gravity. Phys. Rev. D 2012, 86, 044005. [CrossRef]

27. Modesto, L.; Rachwal, L. Super-renormalizable and finite gravitational theories. Nucl. Phys. B 2014, 889, 228. [CrossRef]

28. Stelle, K.S. Renormalization of higher derivative quantum gravity. Phys. Rev. D 1977, 16, 953. [CrossRef]

29. Dimitrijevic, I.; Dragovich, B.; Grujic, J.; Rakic, Z. On modified gravity. Springer Proc. Math. Stat. 2013, 36, 251-259.

30. Dimitrijevic, I.; Dragovich, B.; Grujic, J.; Rakic, Z. New cosmological solutions in nonlocal modified gravity. Rom. Journ. Phys. 2013, 58, 550-559.

31. Dimitrijevic, I.; Dragovich, B.; Grujic, J.; Rakic, Z. Some power-law cosmological solutions in nonlocal modified gravity. Springer Proc. Math. Stat. 2014, 111, 241-250.

32. Dimitrijevic, I.; Dragovich, B.; Grujic, J.; Koshelev, A.S.; Rakic, Z. Cosmology of non-local $f(R)$ gravity. Filomat 2019, 33, 1163-1178. [CrossRef]

33. Dimitrijevic, I.; Dragovich, B.; Stankovic, J.; Koshelev, A.S.; Rakic, Z. On nonlocal modified gravity and its cosmological solutions. Springer Proc. Math. Stat. 2016, 191, 35-51.

34. Dimitrijevic, I.; Dragovich, B.; Grujic, J.; Rakic, Z. Some cosmological solutions of a nonlocal modified gravity. Filomat 2015, 29, 619-628. [CrossRef]

35. Dimitrijevic, I. Cosmological solutions in modified gravity with monomial nonlocality. Appl. Math. Comput. 2016, 285, 195-203. [CrossRef]

36. Dimitrijevic, I.; Dragovich, B.; Rakic, Z.; Stankovic, J. On nonlocal gravity with constant scalar curvature. Publ. De L'Institut Math. Nouv. Série 2018, 103, 53-59. [CrossRef]

37. Dimitrijevic, I.; Dragovich, B.; Koshelev, A.S.; Rakic, Z.; Stankovic, J. Cosmological solutions of a nonlocal square root gravity. Phys. Lett. B 2019, 797, 134848. [CrossRef] 
38. Dimitrijevic, I.; Dragovich, B.; Rakic, Z.; Stankovic, J. Variations of infinite derivative modified gravity. Springer Proc. Math. Stat. 2018, 263, 91-111.

39. Aghanim, N.; Akrami, Y.; Ashdown, M.; Aumont, J.; Baccigalupi, C.; Ballardini, M. Planck 2018 results. VI. Cosmological parameters, Planck collaboration. arXiv 2018, arXiv:1807.06209.

(C) 2020 by the authors. Licensee MDPI, Basel, Switzerland. This article is an open access article distributed under the terms and conditions of the Creative Commons Attribution (CC BY) license (http:// creativecommons.org/licenses/by/4.0/). 